\title{
Systems Biology and Machine Learning in Plant-Pathogen Interactions
}

\author{
Bharat Mishra, ${ }^{1}$ Nilesh Kumar, ${ }^{1}$ and M. Shahid Mukhtar ${ }^{1,2, \dagger}$ \\ ${ }^{1}$ Department of Biology, and ${ }^{2}$ Nutrition Obesity Research Center, University of Alabama at Birmingham, 1300 University Blvd., \\ Birmingham 35294, U.S.A.
}

Accepted 16 October 2018.

Systems biology is an inclusive approach to study the static and dynamic emergent properties on a global scale by integrating multiomics datasets to establish qualitative and quantitative associations among multiple biological components. With an abundance of improved high throughput -omics datasets, network-based analyses and machine learning technologies are playing a pivotal role in comprehensive understanding of biological systems. Network topological features reveal most important nodes within a network as well as prioritize significant molecular components for diverse biological networks, including coexpression, protein-protein interaction, and gene regulatory networks. Machine learning techniques provide enormous predictive power through specific feature extraction from biological data. Deep learning, a subtype of machine learning, has plausible future applications because a domain expert for feature extraction is not needed in this algorithm. Inspired by diverse domains of biology, we here review classic systems biology techniques applied in plant immunity thus far. We also discuss additional advanced approaches in both graph theory and machine learning, which may provide new insights for understanding plant-microbe interactions. Finally, we propose a hybrid approach in plant immune systems that harnesses the power of both network biology and machine learning, with a potential to be applicable to both model systems and agronomically important crop plants.

\section{SYSTEMS BIOLOGY AND MACHINE LEARNING IN COMPLEX BIOLOGICAL SYSTEMS}

Systems biology entails a systematic and comprehensive understanding of genetic, molecular, biochemical, physiological, and morphological information of static as well as dynamic biological processes of an organism as a whole (Barabási and Oltvai 2004; Garbutt et al. 2014). This global approach can unravel the emergent properties of a biological system. Toward this end, systems biology necessitates a "top-down" strategy by integrating diverse -omics approaches associated with systems

B. Mishra and N. Kumar contributed equally to this work.

${ }^{\dagger}$ Corresponding author: M. Shahid Mukhtar; E-mail: smukhtar@uab.edu

Funding: This work was supported by the National Science Foundation Directorate for Biological Sciences (IOS-1557796) to M. S. Mukhtar.

*The $\boldsymbol{e}$-Xtra logo stands for "electronic extra" and indicates that one supplementary table is published online.

(c) 2019 The American Phytopathological Society analyses, where qualitative and quantitative datasets of multiple components (genes, RNA, proteins, metabolites, ions, and so on) are measured (Fig. 1) and their relationships are established (Barabási and Oltvai 2004; Garbutt et al. 2014). Moreover, feasible mathematical and computational models are reconstructed, which constitute formal descriptions of the components to illustrate and compute the associations of the systems' layers and describe the local as well as global properties of the system (McCormack et al. 2016; Pritchard and Birch 2014). Subsequently, these models can be experimentally verified to establish novel hypotheses which, in turn, further increase the resolution of designed models. Finally, based on the datasets and the models designed, computational algorithms should be time efficient to expeditiously compute the behavior of complex systems. This ultimately enhances the detailed understanding of a biological system to discover and reconstruct novel biological traits (Barabási and Oltvai 2004; Pritchard and Birch 2014).

Biomolecules and their interactions can be mathematically represented in a mechanistic model (Bordbar et al. 2014; Le Novère 2015). Generally, three types of mechanistic models are used in systems biology based on the interactions between components: (i) constraint-based models, for metabolic reaction networks in biosynthetic pathways, flux balance analysis, and ionomic networks (Schatschneider et al. 2013); (ii) logical models, for large-scale interaction networks that can determine the change of any biological event based on one or more than one components, coexpression networks, gene regulatory networks (posttranscriptional and posttranslational), and protein-protein interaction networks; and (iii) kinetic models: ordinary differential equation-based models that explain the dynamics of a system by determining the concentration kinetics and conditions of cellular constituents by law of mass action and enzyme kinetics (Smallbone et al. 2010). It is also essential to enlist all stages of model building and simulations for verification by the computational systems biology community and to ensure reproducibility. The systems biology community has a few open standard rules that are worth mentioning: Minimal Information Required for the Annotation of Models (MIRIAM) (Le Novère et al. 2005) and Minimal Information About a Simulation Experiment (MIASE) (Waltemath et al. 2011b). Also, standard format encoding is needed for the model design as well as simulations and analyses. Typically, Systems Biology Markup Language (SBML) (Hucka et al. 2003) and Simulation Experiment Description Markup Language (SED-ML) (Waltemath et al. 2011a) are employed. The advancement in automated model generation, share, reuse, and analyses is a huge driver in systems biology (Büchel et al. 2013). 
In parallel, machine learning is a part of artificial intelligence that often uses applied statistical and computational techniques to give the script an ability to "learn" with data, without being programmed. It utilizes diverse patterns of data consisting of "features" and "labels" for predictive modeling (Camacho et al. 2018). Upon learning a set of patterns, the model can predict any range of output. Thus, the type of a biological system, data patterns, and its adequacy (an input for machine learning) are essential for efficient predictions. In biological applications, features can include an array of datasets such as a genomic sequence, gene expression profiles, protein-protein interactions, metabolite concentrations, and so on (Fig. 1). Primarily, machine learning methods are categorized into groups-namely, unsupervised and supervised learning (McMurray and Hollich 2009). Although the former method entails unknown features, the latter approach is applied to known labels or features. In the supervised case, the known features are used to train the machine-learning model prior to making the predictive model (Camacho et al. 2018).

Characteristically, diverse plants' biological processes, including growth and development under different physiological conditions, are increasingly complex. For instance, to survive in often quite extreme environments, plants have a tendency to adapt toward any biotic and abiotic stress (Becklin et al. 2016). In biotic stresses, pathogenic microorganisms (bacteria, viruses, oomycetes, and fungi) are highly studied factors that alter host fitness levels as a consequence of pathogenesis (Afzal et al. 2016; Kamoun et al. 2015; Lopez et al. 2015). Additionally, host plants and pathogenic microbes have competitively evolved and acquired numerous bimolecular apparatuses that have the potential to modulate the possible outcome of their interactions (Abdullah et al. 2017). Furthermore, different
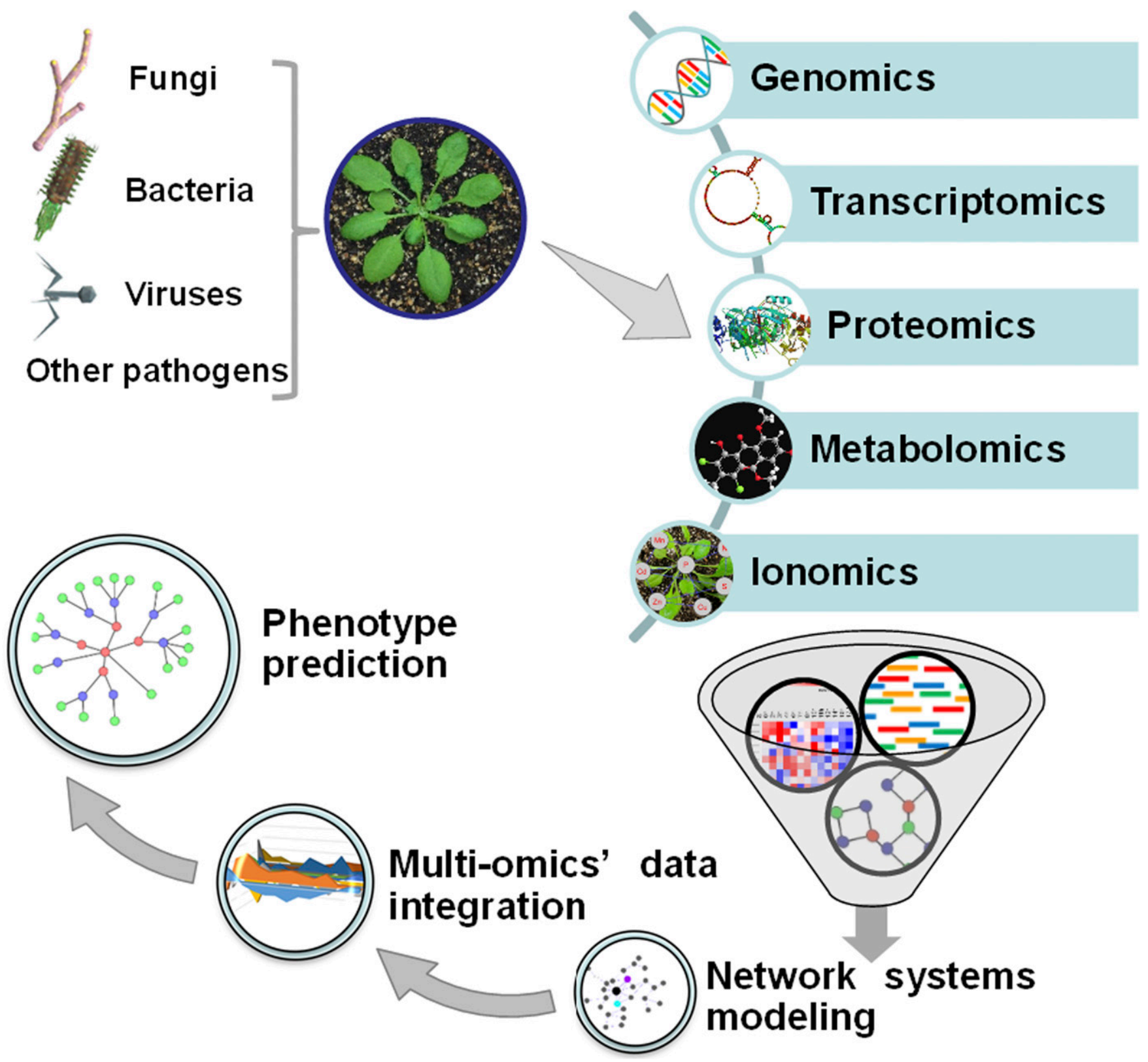

Fig. 1. Multiomics data integration and network systems modeling for comprehensive understanding of diverse plant pathosystems. Infection of diverse phytopathogens, including fungi, bacteria, and viruses, to Arabidopsis is shown. Global changes in various -omes in both plants and pathogens are demonstrated. Identification of potential immune- and susceptibility-related modules and genes can be achieved through multiomics data integration by networksystems modeling approaches, including clustering, predictive modeling, and pairwise data integration. 
abiotic environmental factors, including temperature and light, also affect plants' response to pathogenic microbes (Huot et al. 2017). Thus, plant-pathogen interactions constitute an intricate and multilayered network involving molecular players from both virulent microbes and their hosts (Mukhtar et al. 2016; Abdullah et al. 2017). Phytopathogens utilize a suite of diverse molecules, including nucleic acids and proteins (hereafter called effectors) for a successful infection, known as effectortriggered susceptibility (ETS). In contrast, plants employ their immune systems to detect molecular components of the pathogens and trigger robust defense responses, including microbial-associated molecular pattern immunity (MTI) or effector-triggered immunity (ETI) (Mukhtar 2013, 2016; Washington et al. 2016). These diverse immune responses require phytohormonal signaling cascades involving both positive and negative transcriptional outputs (Tsuda 2018). Intriguingly, recent transcriptome analyses of both plants and pathogens indicate that host susceptibility or immune outcomes shape pathogens' transcriptional regulatory networks (Lovelace et al. 2018; Nobori et al. 2018b; Westermann et al. 2012; Wolf et al. 2018). Toward this end, a conceptual framework was proposed highlighting the molecular interactions of networks between plant and pathogens, as reviewed by Nobori et al. (2018a). Therefore, systems biology and machine learning have huge implications in multiomics data integration, data analytics, and multidimensional network modeling pertinent to plantpathogen interactions to predict the outcome of a given scenario (Fig. 1). In this review, we will discuss the recent advances and future perspective in network biology and machine learning techniques with respect to the studies of plant immunity and pathogens' infection.

\section{NETWORK BIOLOGY AND DIVERSE CENTRALITY MEASURES IN PLANT-MICROBE INTERACTIONS}

To generate any type of cellular response for a desired functional outcome, there are different types of network components constructive to cellular functions. This includes gene coexpression, protein-DNA interactions, noncoding RNA (ncRNA)-DNA interaction, ncRNA-protein interaction, proteinprotein interaction, and metabolite reaction networks (Fig. 1). The functional modules embedded within these networks translate the flow of information under different environmental and cellular conditions (Garbutt et al. 2014). In contrast, pathogenic molecular components interfere with these modules and rewire the flow of information for their benefit (Mukhtar et al. 2011; Pajerowska-Mukhtar et al. 2013). To provide comprehensive and systematic understanding of these cross-species interactions, network biology-based analyses are pivotal (Pritchard and Birch 2014). These network analyses can reveal genotype-phenotype relationships by exploring the complex layers of biological systems (Ahmed et al. 2018). Akin to any social, physical, electrical, and communication networks, biological systems possess interacting components termed "nodes". Nodes in these systems are interconnected based on their physical or functional relationships, defined as edges or links. These interactions follow directed or undirected representation and depend upon interaction type (i.e., one to one, one to many, many to one, and many to many relationships), which can define network topology (McCormack et al. 2016). Structural centralities within a network can be divided into three groups based on the topological features used: (i) neighborhood-based centralities measure the influence and behavior of its surrounding neighbors such as degree, LocalRank, ClusterRank, coreness, and H-index; (ii) path-based centralities measure the distance between two nodes such as shortest path length, eccentricity, closeness centrality,
Katz centrality, information centrality, and betweenness centrality (Gräßler et al. 2012); and (iii) iterative refinement centralities measure the mutual enhancement effect of a given node by determining the number of neighbors and their influence in the network like eigenvector centrality, cumulative nomination, PageRank, LeaderRank, and stochastic approach for link structure analysis (SALSA) (Lü et al. 2016a). Methodologies and concepts of these centrality measures are provided in Figure 2 and Supplementary Table S1. Several structural centralities and iterative refinement centralities have been utilized to discover the most important nodes in plant-microbe interactions (Barabási and Oltvai 2004; Lü et al. 2016a).

In both prokaryotes and eukaryotes, most of the networks display scale-free property (i.e., whereas the majority of nodes exhibit fewer connections, a few nodes have more connections, which is referred as a power law distribution of the network) (Pan et al. 2016). These highly connected nodes are known as hubs, while betweenness in the network, another centrality measure, determines the frequency of a node in facilitating interactions with other nodes through shortest paths (Fig. 2). Both hubs and high betweenness (bottlenecks) have been used as an indicator of significant nodes in diverse intra- and interspecies interactions in plants (McCormack et al. 2016). In a recent host-associated microbial community network analysis, a small number of taxa were found to be strongly interconnected. These microbial hubs play major roles in shaping microbial communities on plant hosts (Agler et al. 2016). Degree and betweenness analyses revealed network hubs within a symbiont-symbiont co-occurrence network of diverse plant roots (Toju et al. 2016, 2018). Further network analyses showed that diverse functional groups of fungi are compartmentalized into specific modules (Toju et al. 2016). Such network-based meta-community analyses can determine the specific set of microbial species that can organize the overall root-microbiome dynamics, and have potential agricultural applications. Similarly, these centrality measures have been used in plant coexpression networks to prioritize key factors among thousands of transcripts (Serin et al. 2016). For instance, network topological analyses in Arabidopsis-bacterial coexpression discovered new immune-related players (Jiang et al. 2016; Mishra et al. 2017; Tully et al. 2014). Coexpression network analyses in seven plant species followed by network architecture analyses determined that hubs and bottlenecks negatively correlate with the rate of evolution (Masalia et al. 2017). Moreover, it was shown that hubs are evolutionarily conserved and enriched in intrinsically disordered proteins (Barabási and Oltvai 2004; Rangarajan et al. 2015). Consistent with these findings, hubs and bottlenecks were proposed to be essential nodes in the yeast protein-protein interaction network (interactome), a phenomenon known as centrality-lethality rule (He and Zhang 2006; Yu et al. 2007). However, this network property was not validated in other eukaryotic systems. In a recent comprehensive study, Arabidopsis interactome was integrated with a compendium of over 4,300 Arabidopsis phenotypes to test this network topology feature. In contrast to the centrality-lethality rule, nodes corresponding to hubs and bottlenecks were associated with conditional phenotypes (Ahmed et al. 2018). Regardless of a negative correlation between essentiality and hubs as well as bottlenecks, they remained the most influential nodes within a network, and are shown to be targets of pathogenic virulence factors (effectors) in plants as well as other eukaryotic interactomes (Ahmed et al. 2018; Mukhtar et al. 2011; Pan et al. 2016; Weßling et al. 2014). Because an arbitrary value defines a hub and a bottleneck within a network, Ahmed et al. (2018) elegantly showed that proteins corresponding to hubs as well as bottlenecks at various thresholds display significant enrichment in effector targets. Consistently, hubs and bottlenecks were 
associated with conditional and immune-related phenotypes (Ahmed et al. 2018).

Although hubs and bottlenecks have an extraordinary potential to predict effector targets in Arabidopsis and other eukaryotic interactomes, their limitation is that these two centrality measures constitute only 5 to $10 \%$ of nodes within the network. This makes the predictive power of effector targets very low (e.g., approximately $6.5 \%$ in the Arabidopsispathogen interactome (Ahmed et al. 2018). In addition to these network centrality measures, another method, termed $k$-shell (or $k$-core) decomposition, is implemented in social networks to identify the most influential information spreaders (Pei et al. 2014). The $k$-shell decomposition method systematically removes or decomposes shells or layers of a network to identify core nodes in network connectivity (Fig. 2). A modified version of the $k$-shell decomposition known as weighted $k$-shell decomposition was recently implemented to study effector discovery rate in two major Arabidopsis interactomes, and that increased the success rate up to $40 \%$ (Ahmed et al. 2018). Moreover, in two recent studies, a novel approach termed "information centrality method" was used to explore the flow of information between nodes and significant information

\section{A}

Hub = high Degree

$$
\text { Degree }\left(k_{i}\right)=\sum_{j} a_{i j}
$$

where, $a_{i j} \geq 0$

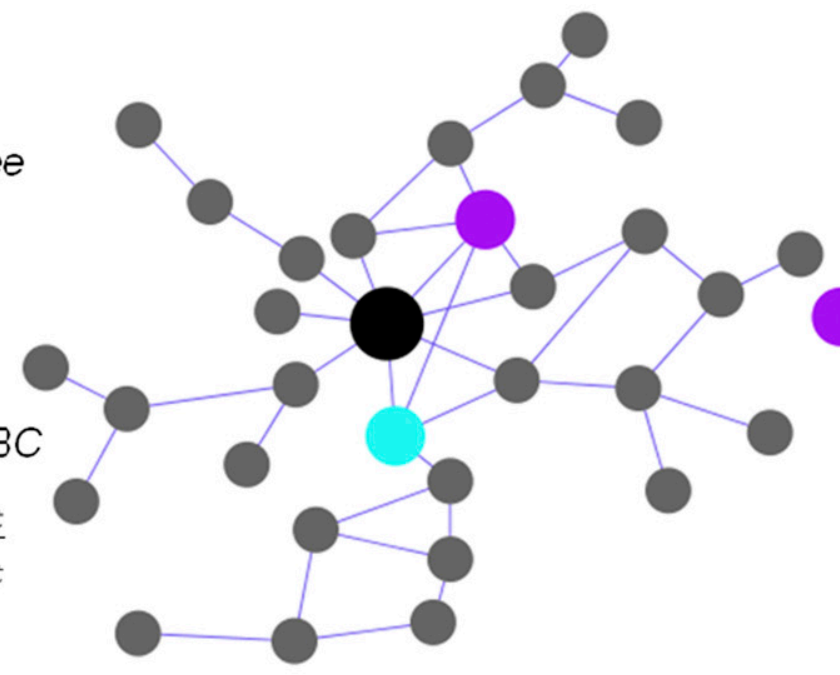

Eigenvector centrality

$E C\left(x_{i}\right)=c \sum_{j=1}^{n} a_{i j} x_{j}$

where, $E C\left\{x_{i}\right\}=0$ to 1

B

Weighted $k$-shell

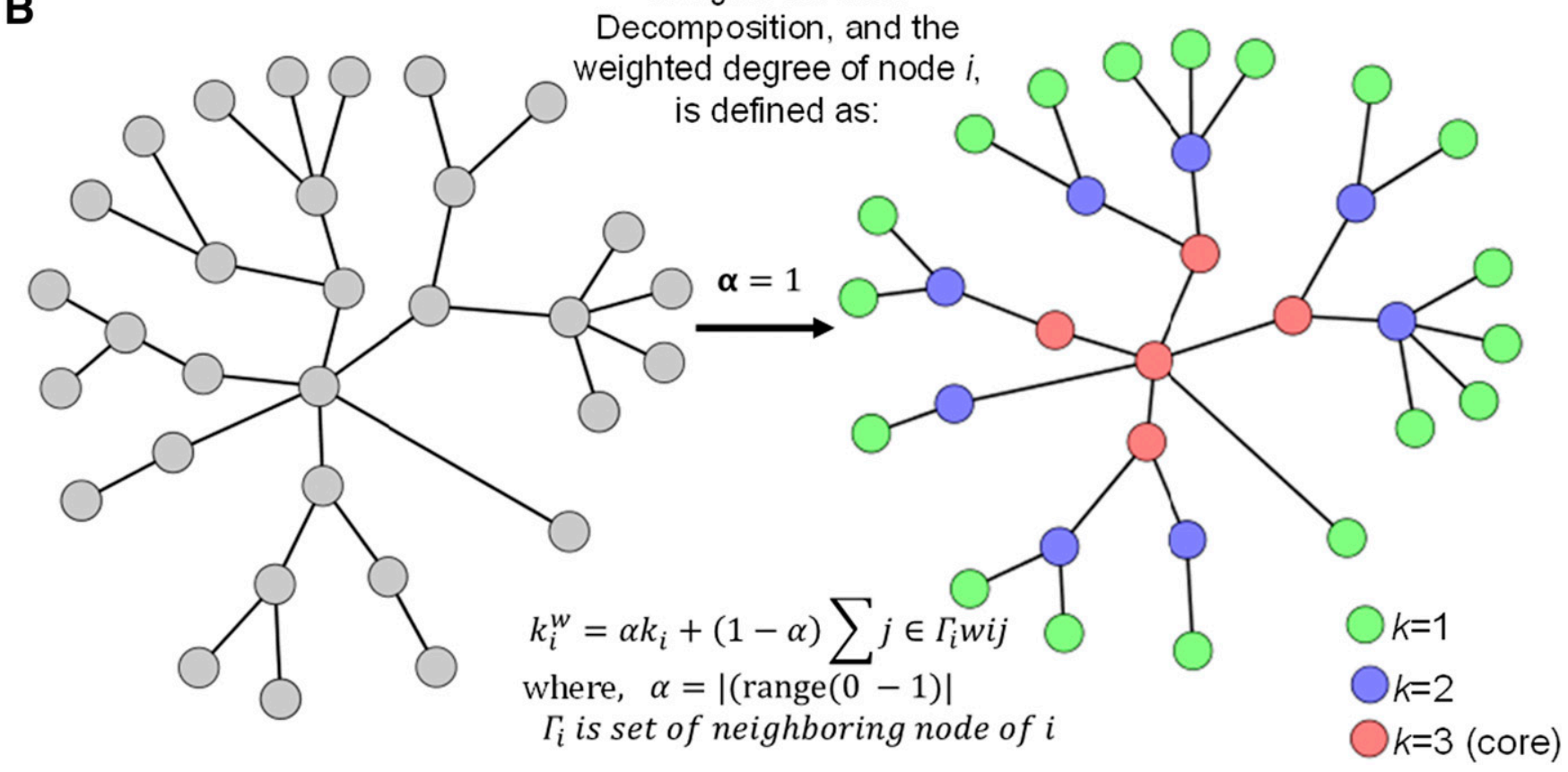

Fig. 2. Biological scale-free network topology represented by nodes (vertices) and edges (links) in a directed or undirected network. A, Graphical representation of centrality measures were shown in a hypothetical network. Highly connected nodes (hubs) are shown in black. Bottleneck is a path-based centrality measure with high betweenness centrality node determined as the bridge between two subnetworks represented in the aqua node. Degree and betweenness centrality are different types of structural centralities. Eigenvector centrality is an iterative refinement centrality measure determined by the number of neighbors and influence of each neighbor on the given node represented in the purple node. B, Weighted k-shell decomposition method is an improved k-shell decomposition based on the coreness of the connected network. The left panel represents the hypothetical network with gray nodes, while the right panel represents the hypothetical network decomposed into three shells $(k)$. The value of $k=1$ represents the outermost layer with nonsignificant nodes (green), $k=3$ represents the innermost layer (core) of the network with most significant nodes (pink), while $k=2$ represents the periphery of the core with somewhat significant nodes (blue). 
spreaders, which was previously limited by the use of betweenness centrality method (Ahmed et al. 2018; Mishra et al. 2017). Information centrality measures the flow of information in the largest connected component of the network by quantifying the information flow through different paths between two proteins. Effector targets exhibit heightened information centrality compared with nodes located in the periphery of the network or noneffector targets. Taken together, it was proposed that Arabidopsis utilizes hubs and bottlenecks to spread information through differentially expressed genes (DEG), while pathogens interact with these most influential nodes to rewire the flow of information (Ahmed et al. 2018; Mishra et al. 2017). Finally, several of the studied centrality measures were shown to be significant predictors of key players in the plant immune system.

\section{POTENTIAL IMPLICATIONS OF NOVEL NETWORK STRUCTURAL CENTRALITIES IN PHYTOPATHOLOGY}

The aforementioned network-centric centrality methods are advantageous in the modeling of plant-microbe interactions. The next question is whether additional structural centrality measures can be utilized to decipher the network topology features in disease- or pathogen-affected networks as well as allow for significant gene prioritization (Barabási and Oltvai 2004; Das et al. 2018; Lü et al. 2016b). Several such topological features have been employed in different eukaryotic systems. For instance, clustering coefficient (the geometric average of the normalized edge weights of subgraph) has been utilized in several plant coexpression networks. This includes five plant pathosystem coexpression networks to discover common and distinct immune signaling pathways among Arabidopsis, rice, soybean, tomato, and cassava (Leal et al. 2014). In parallel, clustering coefficient based on a double label propagation clustering algorithm was used in a coexpression network analysis in the study of Huntington's disease. This study identifies nine disease-related modules with maximum likelihood to be involved in the pathogenic infection (Jiang et al. 2017). Integration of various layers of -omics is vital to decipher genotype-phenotype relationships (Garbutt et al. 2014). In such an analysis, the shortest path length between source and target nodes was measured by integrating Arabidopsis interactome and plant immune-related transcriptomics data. It was demonstrated that the path lengths between effector targets and DEG are smaller than that of non-DEG, suggesting that the information from effector targets travels faster to DEG than non-DEG (Mishra et al. 2017). Furthermore, integration of MTI- and ETS-dependent time-course transcriptome datasets with the Arabidopsis interactome led to the discovery of stable and temporal disease resistance-related as well as disease susceptibility-related complexes (Mishra et al. 2017), an area of dynamic protein-protein interactions that is not fully explored due to the inadequacy of current detection technologies. Likewise, a disease-autophagy network was generated in humans by incorporating known disease and autophagy genes as well as protein-protein interactions. Shortest-path analysis determined 11 different disease classes involving cancer, metabolic diseases, and hematological diseases that overlap with autophagy-related genes (Wang et al. 2017). PageRank, an algorithm that is employed by Google Search to rank websites, was used to predict disease-related genes by integrating tissue specific gene expression data and protein-protein interactions to generate a tissue-specific network along with DNA methylation for accurate identification of the top 20 colon cancer- and leukemia-related genes ( $\mathrm{Li}$ et al. 2014). Similarly, PageRank used to study the connectivity influence of extracellular domains of receptor-like kinases in Arabidopsis cell surface interactome. This analysis revealed BRI1associated receptor kinase 1 (a functional hub) as the most connected node (structural hub) (Smakowska-Luzan et al. 2018). In another gene prioritization study, LocalRank measure was used in a human interactome that was integrated with functional linkage networks to find the most important genes by also considering the fourth-order neighbors for each gene that are not generally captured by degree of genes in different human diseases (Meshkin et al. in press). Whether LocalRank could yield novel disease genes in Arabidopsis-pathogen and other plant-pathogen interactomes is an area of future research.

A broad spectrum of other network topology features has been used in human and other eukaryotic networks. Here, we featured a selection of such network centrality measures that have the potential to be implemented in diverse networks of plant-microbe interactions. In a multiomics integrative study, coexpression, protein-protein interaction, and functional interaction networks were incorporated, followed by network topology analyses. Not only the immediate neighbors of a node as described for degree centrality but also the locations of the nodes within a network are equally important for the identification of most influential nodes. Such network location-based centralities include structural holes and coreness. To establish phenotype-gene association in breast cancer, structural holes, coreness, and degree predicted significant genes more accurately than any other centrality measures (Ramadan et al. 2016). Given that hubs and betweenness are associated with oncogenesis as well as targets of pathogen effectors and microbial hubs, these novel centrality analyses would possibly provide insights into plant-microbe interactions. Similarly, eccentricity along with other topological measures were utilized in a human protein-protein interaction network to predict potential novel disease-associated genes and differentiate among disease- and nondisease-associated proteins (Carson and Lu 2015). Moreover, Katz centrality was used for candidate disease-related gene prioritization by integrating 58 gene expression datasets, protein-protein interaction strength, and known disease-related genes in 40 human diseases (Zhao et al. 2011). In another experiment, closeness centrality along with degree, clustering coefficient, stress centrality, and betweenness centrality were used in coexpression network analysis and identified 3 of 13 hub genes in all centrality measures that are conserved in hepatocellular carcinoma (Liu et al. 2016). Similarly, such centrality measures, including $\mathrm{H}$-index, LocalRank, Katz centrality, cumulative nomination, LeaderRank, SALSA, and so on, can be utilized in plant biotic stresses and disease resistance as well as plant-microbe interactions to discover emergent network components. In sum, the systematic deciphering of network design and comprehensive understanding of the topological properties of a biological network may serve as a crucial step in the breakthrough identification of novel modules in a complex biological system, which can deliver experimentally testable propositions.

\section{MACHINE LEARNING, A DATA-ANALYTICAL PREDICTIVE MODELING PLATFORM IN DIVERSE BIOLOGICAL SYSTEMS}

Machine learning exhibits wider applications, including disease prognosis in humans and agriculture, weather forecasting, stock exchange, and e-commerce, by using social networking data (Fig. 3). For instance, insect vectors pack a "double whammy" in agricultural crop production worldwide by directly damaging the crop and successfully transmitting infectious pathogens. Random forests, Hidden Markov Models (HMM), and hierarchical cluster analyses were used to learn 
the feeding patterns of the Asian citrus psyllid that causes citrus greening disease in a recent study (Willett et al. 2016). Similarly, support vector machines (SVM) were trained to predict the transmission patterns of influenza virus (Qiang et al. 2018). Thus, such machine learning studies can facilitate novel intervention strategies in the spread of deadly pathogens in agriculture and other systems. Automation in agriculture is the key to feeding the rapidly growing world population. Using drone-based imaging and remote sensing data, a machine learning approach was employed to predict the biomass of grass sward (Viljanen et al. 2018). Likewise, superresolution aerial imaging was performed on 14 crops, including tomato, grape, apple, corn, and soybean, to discover patterns of disease phenotypes. Furthermore, a convolutional neural network (a deep learning approach) was used for the disease classification. Thus, "features or patterns" of field-based phenotyping under diverse climate conditions can be used as input for improved control of plant diseases. Similarly, a combination of fungicides is often more effective to deter the onset of diseases and their progression than single fungicides, while finding the accurate combination is one of the major challenges on its own. Toward this end, SVM was used to optimize the fungicide rate against a maize fungal pathogen, Bipolaris maydis (Wang et al. 2015). Another major threat to cereal, particularly wheat crops

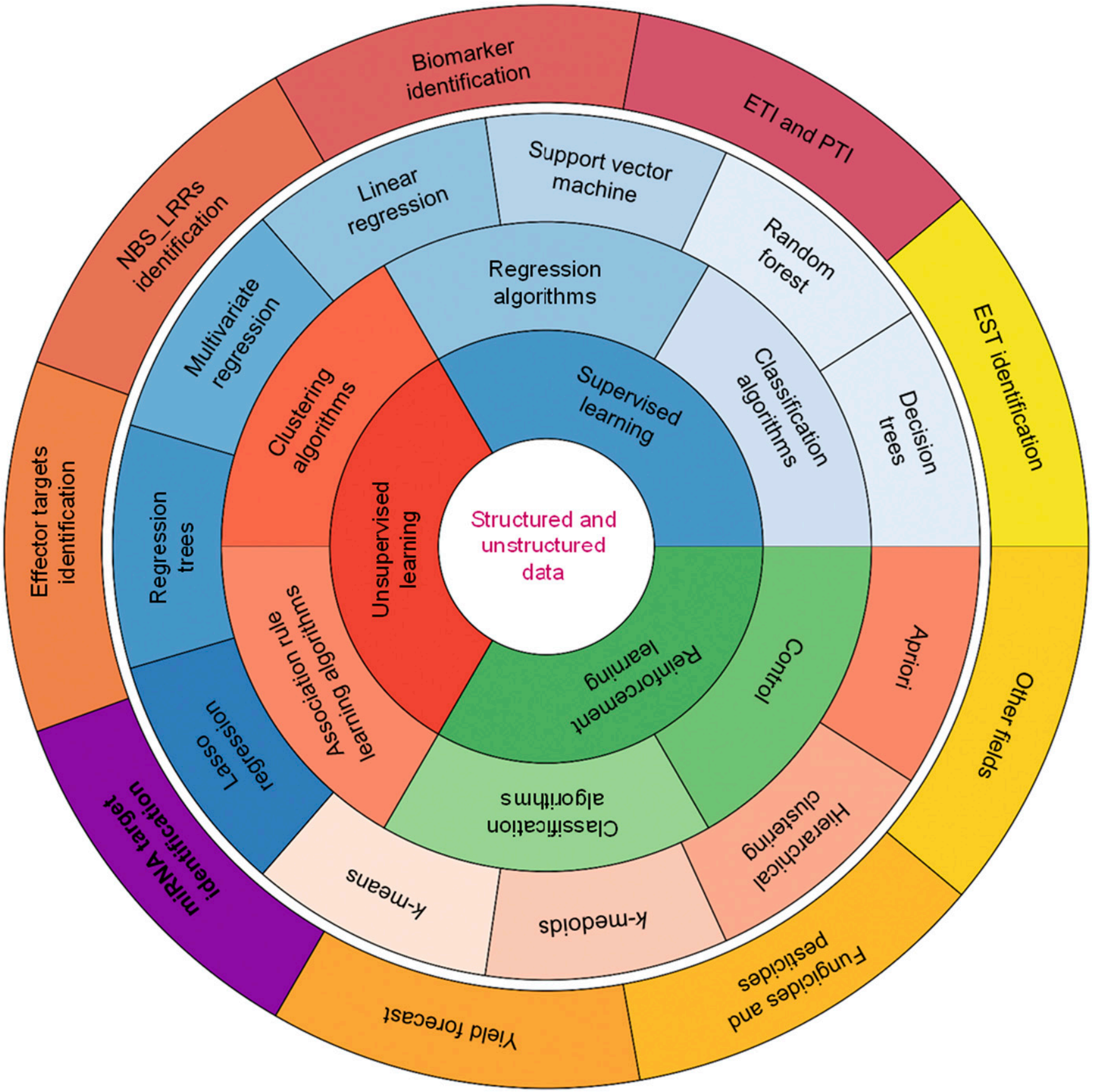

Fig. 3. Different machine learning techniques and their applications. Machine learning can be used on a wide spectrum of structured and unstructured data for the prediction and significant gene prioritization based on three methods (supervised learning, unsupervised learning, and reinforcement learning). Supervised learning includes classification algorithms and regression algorithm techniques: decision trees, random forest, support vector machine, linear regression, multivariate regression, regression trees, and lasso regression methods. Unsupervised learning includes clustering algorithms and association rule learning algorithms: $k$-means, $k$-medoids, hierarchical clustering, and a priori methods. The outermost layer includes the application of these machine learning techniques. 
worldwide, is Stagonospora nodorum blotch (SNB) that reduces up to $50 \%$ of the yield. Because preplanting factors were shown to reduce the severity of SNB, multiple regression and machine learning algorithms-artificial neural networks, categorical and regression trees, and random forests-were used to better predict disease occurrences (Mehra et al. 2016). Thus, machine learning has been employed as a predictive modeling tool in a wide range of systems, including crop production and disease prognosis in field conditions (Fig. 3).

At the molecular level, machine learning enables predictive modeling from large and multidimensional datasets to elucidate the complex relationships between genome and phenome in a biological system (Camacho et al. 2018). It has a wide range of applications in various -omics, including genomics, transcriptomics, interactomics, proteomics, and metabolomics (Camacho et al. 2018; McMurray and Hollich 2009). In genomics, machine learning is employed to recognize the locations of transcription start sites in a genomic sequence (Ohler et al. 2002) and identify splicing sites (Degroeve et al. 2002), enhancers (Bucher 1990), and promotors (Heintzman et al. 2007). Machine learning can also be used for the functional annotations of genes (Ashburner et al. 2000) using genomic sequences, gene expression regarding phenotypes, proteinprotein interaction networks, and transcription factor (TF) binding data as input. It has also been employed to predict gene expression on the basis of the genomic sequence (Beer and Tavazoie 2004), ChIP-seq profiles of histone modification (Karlić et al. 2010), and TF binding (Friedman 2004; Ouyang et al. 2009) or using complex data such as expression networks. Machine learning-based classification methods have been used to analyze microarray data. These methods include SVM, radial basis function neural nets, multilayer perceptron (MLP) neural nets, Bayesian, decision tree, and random forest (Pirooznia et al. 2008). The daunting task of solving protein secondary and tertiary structures was approached by using deep convolutional neural fields (Wang et al. 2016). The tertiary structure problem was addressed through unsupervised clustering methods and supervised machine learning methods, including HMM, neural networks, and SVM for three-dimensional and four-dimensional structure prediction problems (Cheng et al. 2008). The question that still needs to be addressed is how to use these diverse features in plant-microbe interactions (details below). Collectively, machine learning has proven very useful in predictive modeling of a wide range of biological and cellular processes.

\section{UTILIZATION OF MACHINE LEARNING IN MULTIDIMENSIONAL DATASETS IN PLANT-PATHOGEN INTERACTIONS}

For the last two decades, machine learning has been employed in a wide spectrum of plant immunity to discover novel components ranging from the classifications of expressed sequence tags (Friedel et al. 2005) to the identification of novel disease resistance-related biomarkers (Wójcik et al. 2015), as well as the discovery of shared and distinct immune pathways (Dong et al. 2015) (Fig. 3). Nucleotide-binding domain leucinerich repeat (NLR)-containing is one of the biggest families of disease resistance genes that often trigger ETI (Mukhtar 2013). NBSPred, an SVM-based classification model, was developed to differentiate NLR genes among genomic, transcriptomic, and protein sequence data across monocots and dicots (Kushwaha et al. 2016). With the discovery of NLR that possess integrated domains that might have potential decoy and transcriptional roles (Baggs et al. 2017), as well as the availability of unprecedented genomic data from diverse crop plants, machine learning could be implemented to discover novel specifications of NLR with potential genetic engineering programs.
Similarly, receptor-like kinases (often involved in patterntriggered immunity [PTI]) are predicted using HMM and phylogenetic analyses (Brustolini et al. 2017). Another molecular player from hosts and their pathogens, microRNA (miR), plays a wide range of cellular functions in plant immunity and exhibits significant potential for generating disease resistance crop plants (Djami-Tchatchou et al. 2017). However, accurate identification of miR targets is an essential step for a candidate miR to avoid unwanted suppression of off-targets. Toward this end, an integrative machine learning method was used to predict miR target genes, which include disease resistance genes and TF, to get novel insights into plant-pathogen interaction networks (Kurubanjerdjit et al. 2013).

Although genomic and transcriptomic sequences of phytopathogens discover novel components that may physically or functionally interact with the host machinery, characterization of hypothetical genes in pathogens is still not fully understood. Using genomic data from two bacterial pathogens, Dickeya dadantii 3937 and Pectobacterium carotovorum WPP14, a supervised machine learning model discovered over 200 novel factors that have been implicated in host-microbe interaction (Ma et al. 2014). Likewise, accurate prediction of pathogen effectors has been a major challenge. Protein features such as frequencies of amino acids, frequencies of amino acid classes in the sequence, molecular weight and length of the sequence, and protein net charge were used to train the model. Several machine learning algorithms, including Naive Bayes, logistic regression, MLP, C4.5 decision tree, and random forest, were employed for the prediction of fungal effector proteins. This led to the discovery of the first fungal effector prediction tool, EffectorP (Sperschneider et al. 2016). Using a similar approach, ApoplastP was recently developed to reveal aploplastic effectors (Sperschneider et al. 2018). In an effort to identify the recognized components of pathogens (avirulence factors), machine learning approach was used to unravel novel pathogenic specificities (Figueroa et al. 2016). Prediction of effector targets in the host still remains a daunting task. Although several network topological properties have been used to decipher the properties of effector targets, machine learning algorithms, Naive Bayes, and logistic regression classifiers were used for the discovery of transcription activator-like effector targets in bacterial leaf streak of rice (Cernadas et al. 2014). Although ETI and PTI are the two most studied plant immune responses, the global understanding of their signals as well as downstream cascades remains unclear (Dong et al. 2015). A combination of machine learning (network-guided forest) and graph theory has been used to construct network models for both types of plant immunity to explain the quantitative differences in the global network architecture. In this hybrid approach, plant immune coexpression, TF-target, and protein-protein interaction networks were assembled. Comparative analyses between highly significant 1,856 PTI genes and 1,843 ETI genes discovered common and distinct patterns of signaling components (Dong et al. 2015). Thus, prediction of effectors and their targets as well as specific immune responses by using machine learning is a step forward in devising new agricultural strategies against deadly pathogens.

\section{A HYBRID APPROACH: INTERSECTION OF NETWORK BIOLOGY AND MACHINE LEARNING TO UNRAVEL COMPLEXITIES OF PLANT-PATHOGEN INTERACTIONS}

Elucidating how complex phytopathogen infection arises from diverse plants and their pathogens is the primary challenge in agricultural systems. As we deal with multilayered and large-scale -omics data, a hybrid approach that harnesses the 
power of both machine learning and network biology may provide new insights into understanding genes to phenotype relationships (Karr et al. 2012, 2014). As discussed above, the progress thus far made in comprehending intricate relationships between plants and their pathogens at both molecular and fieldcondition levels is based on the applications of network topology analyses or machine learning approaches. As we continuously generate massive amounts of high accuracy data, we may need more advanced learning algorithms to infer better outcomes. One such promising algorithm is deep learning (Bengio et al. 2013; LeCun et al. 2015; Schmidhuber 2015). Although the traditional machine learning approach relies on labor-intensive preprocessing engineering pipelines and data transformation that utilizes visible feature extraction, deep learning autonomously learns characteristics or patterns and utilizes a cascade of multiple layers of nonlinear processing units for feature extraction (Fig. 4). Moreover, deep learning can efficiently tackle multiple object detection problems compared with the known machine learning algorithms such as SVM. Finally, deep learning performs better with large-volume data (Bengio et al. 2013; LeCun et al. 2015; Schmidhuber 2015) (Fig. 4). However, these novel approaches equally draw numerous challenges. The first and foremost constraint is the limited collection of well-annotated large-scale multiomics plant immune datasets. The second aspect is concerned with the integration of multiomics as well as data analyses using network approaches.

Toward this end, efforts are already being made to generate high-quality, large-scale multiomics datasets pertinent to plant pathology. This includes plant immune coexpression networks and phytopathogen-mediated TF-target-directed networks, as well as plant-pathogen physical interaction networks (Ahmed et al. 2018; McCormack et al. 2016; Mishra et al. 2017). In parallel, network biology is yet another great tool that can solve

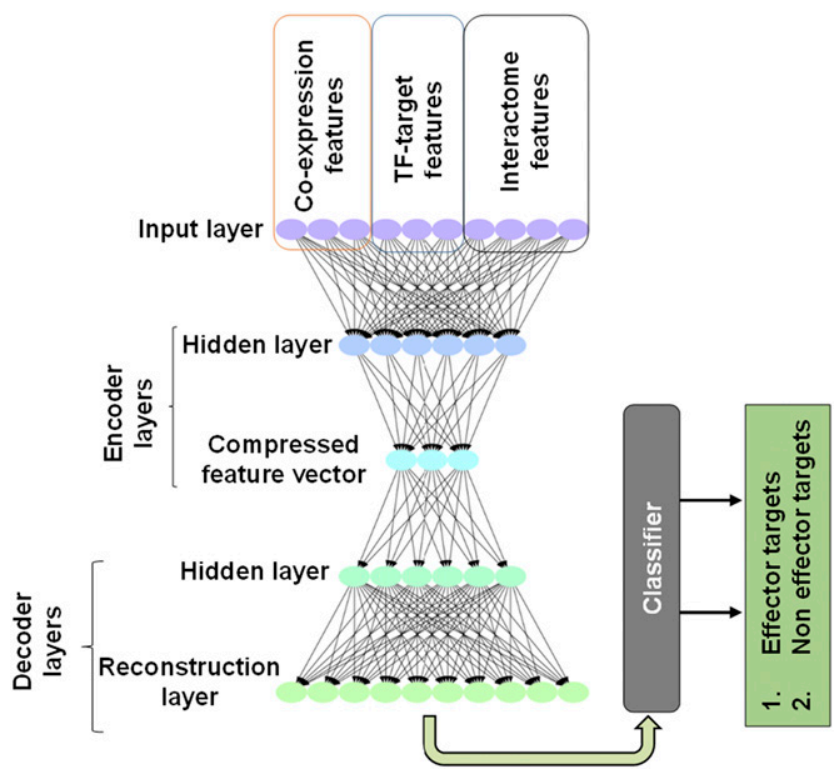

Fig. 4. Schematic representation of deep learning algorithm having the ability to learn features autonomously. The input layer can have different biological datasets as network (coexpression, transcription factor [TF]target, or interactome). Deep learning uses Autoencoder to learn the features from the input layer using encoder and decoder, the most important part of the deep learning algorithm. The encoder layer extracts features and then compresses them for dimensional reduction. Then, the decoder layer performs the reconstruction and, finally, decodes the features. The output layer could have different layers representing each class, including effector targets or noneffector targets, disease or no disease, and genotypephenotype association. problems associated with complex networks or graphs via the discovery of topological features. Toward this end, a wide spectrum of algorithms and computational tools has been used to extract hidden features of a network (Hagberg et al. 2008). These features could be connectivity, $k$-component, smallworld property, clustering, dominating sets, Ramsey (maximum cliques and in depended sets), vertex set, bridges, entropy, communicability, partition, transitivity, constraints, and rich clubs. An important connectivity feature in a network is the identification of hubs (party and date hubs), which allow us to decipher the interactions of genes and their products under diverse physiological and environmental conditions (Chang et al. 2013). In conclusion, several parameters of centrality measurements may act as indicators of "influential nodes" or "super-spreader of information" in a given network and could be used to map raw inputs to feature at the encoder layer. Furthermore, decoder layer takes this feature representation as input, processes it to make its decision, and produces an output. This is called an encoder-decoder network in deep learning (Fig. 4).

Thus, a hybrid approach combining network topology and machine learning would allow us to decode how a gene or a set of genes and their products participate in a plant-pathogen interaction (Fig. 4). Moreover, it will also reveal how a set of genes affecting a disease resistance or a susceptibility phenotype operates in different molecular pathways (Brodie et al. 2014).

\section{CONCLUSIONS}

In summary, to study and interpret molecular biology events in a biological system, a wide range of -omics-based approaches has been meaningfully applied. These comprehensive and "big data" studies generated lots of excitement in the discipline of plant-microbe interactions. To establish a meaningful relationship between genotype and phenotype, systems and network biology have been applied. Network biology, extensively discussed above, has provided novel insights into this complex system. In parallel, machine learning has been extensively used to predict new components in plants and pathogens. The hybrid approach of combining deep learning and network centrality features that are derived from multidimensional -omics data may allow us to uncover uncharted territories in plant-microbe interactions. Aforementioned network biology and machine learning techniques can be transcribed from Arabidopsis to a wide spectrum of crop plants to improve agricultural production. Also, these techniques can be applied to predict the strategies in the transmission of diverse pathogens, disease prognosis, pattern of disease phenotypes, and abiotic components such as heat, drought, salinity, and so on in agricultural crops. Furthermore, the prediction and significant gene prioritization for molecular biology experiments in a controlled environment could accelerate their immediate application to different field and ecological conditions.

\section{ACKNOWLEDGMENTS}

We thank K. Mukhtar and T. Detchemendy for critical reading of the manuscript.

\section{LITERATURE CITED}

Abdullah, A. S., Moffat, C. S., Lopez-Ruiz, F. J., Gibberd, M. R., Hamblin, J., and Zerihun, A. 2017. Host-multi-pathogen warfare: Pathogen interactions in co-infected plants. Front. Plant Sci. 8:1806.

Afzal, Z., Howton, T. C., Sun, Y., and Mukhtar, M. S. 2016. The roles of aquaporins in plant stress responses. J. Dev. Biol. 4:9. 
Agler, M. T., Ruhe, J., Kroll, S., Morhenn, C., Kim, S. T., Weigel, D., and Kemen, E. M. 2016. Microbial hub taxa link host and abiotic factors to plant microbiome variation. PLoS Biol. 14:e1002352.

Ahmed, H., Howton, T. C., Sun, Y., Weinberger, N., Belkhadir, Y., and Mukhtar, M. S. 2018. Network biology discovers pathogen contact points in host protein-protein interactomes. Nat. Commun. 9: Article 2312 .

Ashburner, M., Ball, C. A., Blake, J. A., Botstein, D., Butler, H., Cherry, J. M., Davis, A. P., Dolinski, K., Dwight, S. S., Eppig, J. T., Harris, M. A., Hill, D. P., Issel-Tarver, L., Kasarskis, A., Lewis, S., Matese, J. C., Richardson, J. E., Ringwald, M., Rubin, G. M., and Sherlock, G.; The Gene Ontology Consortium. 2000. Gene ontology: Tool for the unification of biology. Nat. Genet. 25:25-29.

Baggs, E., Dagdas, G., and Krasileva, K. V. 2017. NLR diversity, helpers and integrated domains: Making sense of the NLR IDentity. Curr. Opin. Plant Biol. 38:59-67.

Barabási, A. L., and Oltvai, Z. N. 2004. Network biology: Understanding the cell's functional organization. Nat. Rev. Genet. 5:101-113.

Becklin, K. M., Anderson, J. T., Gerhart, L. M., Wadgymar, S. M., Wessinger, C. A., and Ward, J. K. 2016. Examining plant physiological responses to climate change through an evolutionary lens. Plant Physiol. 172:635-649.

Beer, M. A., and Tavazoie, S. 2004. Predicting gene expression from sequence. Cell 117:185-198.

Bengio, Y., Courville, A., and Vincent, P. 2013. Representation learning: A review and new perspectives. IEEE Trans. Pattern Anal. Mach. Intell. 35: 1798-1828.

Bordbar, A., Monk, J. M., King, Z. A., and Palsson, B. O. 2014. Constraintbased models predict metabolic and associated cellular functions. Nat. Rev. Genet. 15:107-120.

Brodie, A., Tovia-Brodie, O., and Ofran, Y. 2014. Large scale analysis of phenotype-pathway relationships based on GWAS results. PLoS One 9: e100887.

Brustolini, O. J., Silva, J. C., Sakamoto, T., and Fontes, E. P. 2017. Bioinformatics analysis of the receptor-like kinase (RLK) superfamily. Methods Mol. Biol. 1578:123-132.

Büchel, F., Rodriguez, N., Swainston, N., Wrzodek, C., Czauderna, T. Keller, R., Mittag, F., Schubert, M., Glont, M., Golebiewski, M., van Iersel, M., Keating, S., Rall, M., Wybrow, M., Hermjakob, H., Hucka, M., Kell, D. B., Müller, W., Mendes, P., Zell, A., Chaouiya, C., SaezRodriguez, J., Schreiber, F., Laibe, C., Dräger, A., and Le Novère, N. 2013. Path2Models: Large-scale generation of computational models from biochemical pathway maps. BMC Syst. Biol. 7:116.

Bucher, P. 1990. Weight matrix descriptions of four eukaryotic RNA polymerase II promoter elements derived from 502 unrelated promoter sequences. J. Mol. Biol. 212:563-578.

Camacho, D. M., Collins, K. M., Powers, R. K., Costello, J. C., and Collins, J. J. 2018. Next-generation machine learning for biological networks. Cell 173:1581-1592.

Carson, M. B., and Lu, H. 2015. Network-based prediction and knowledge mining of disease genes. BMC Med. Genomics 8:S9.

Cernadas, R. A., Doyle, E. L., Niño-Liu, D. O., Wilkins, K. E., Bancroft, T., Wang, L., Schmidt, C. L., Caldo, R., Yang, B., White, F. F., Nettleton, D., Wise, R. P., and Bogdanove, A. J. 2014. Code-assisted discovery of TAL effector targets in bacterial leaf streak of rice reveals contrast with bacterial blight and a novel susceptibility gene. PLoS Pathog. 10: e1003972.

Chang, X., Xu, T., Li, Y., and Wang, K. 2013. Dynamic modular architecture of protein-protein interaction networks beyond the dichotomy of 'date' and 'party' hubs. Sci. Rep. 3: Article 1691.

Cheng, J., Tegge, A. N., and Baldi, P. 2008. Machine learning methods for protein structure prediction. IEEE Rev. Biomed. Eng. 1:41-49.

Das, K., Samanta, S., and Pal, M. 2018. Study on centrality measures in social networks: A survey. Soc. Netw. Anal. Min. 8:13.

Degroeve, S., De Baets, B., Van de Peer, Y., and Rouzé, P. 2002. Feature subset selection for splice site prediction. Bioinformatics 18:S75-S83.

Djami-Tchatchou, A. T., Sanan-Mishra, N., Ntushelo, K., and Dubery, I. A 2017. Functional roles of microRNAs in agronomically important plants-Potential as targets for crop improvement and protection. Front. Plant Sci. 8:378.

Dong, X., Jiang, Z., Peng, Y. L., and Zhang, Z. 2015. Revealing shared and distinct gene network organization in Arabidopsis immune responses by integrative analysis. Plant Physiol. 167:1186-1203.

Figueroa, M., Upadhyaya, N. M., Sperschneider, J., Park, R. F., Szabo, L. J., Steffenson, B., Ellis, J. G., and Dodds, P. N. 2016. Changing the game: Using integrative genomics to probe virulence mechanisms of the stem rust pathogen Puccinia graminis f. sp. tritici. Front. Plant Sci. 7:205
Friedel, C. C., Jahn, K. H., Sommer, S., Rudd, S., Mewes, H. W., and Tetko, I. V. 2005. Support vector machines for separation of mixed plantpathogen EST collections based on codon usage. Bioinformatics 21: 1383-1388.

Friedman, N. 2004. Inferring cellular networks using probabilistic graphical models. Science 303:799-805.

Garbutt, C. C., Bangalore, P. V., Kannar, P., and Mukhtar, M. S. 2014 Getting to the edge: Protein dynamical networks as a new frontier in plant-microbe interactions. Front. Plant Sci. 5:312.

Gräßler, J., Koschützki, D., and Schreiber, F. 2012. CentiLib: Comprehensive analysis and exploration of network centralities. Bioinformatics 28 1178-1179.

Hagberg, A., Swart, P., and Chut, D. S. 2008. Exploring network structure, dynamics, and function using NetworkX. Pages 11-15 in: Proc. 7th Python Sci. Conf. (SciPy2008), Pasadena, CA, U.S.A. G. Varoquaux, T. Vaught, and J. Millman, eds.

He, X., and Zhang, J. 2006. Why do hubs tend to be essential in protein networks? PLoS Genet. 2:e88.

Heintzman, N. D., Stuart, R. K., Hon, G., Fu, Y., Ching, C. W., Hawkins, R. D., Barrera, L. O., Van Calcar, S., Qu, C., Ching, K. A., Wang, W. Weng, Z., Green, R. D., Crawford, G. E., and Ren, B. 2007. Distinct and predictive chromatin signatures of transcriptional promoters and enhancers in the human genome. Nat. Genet. 39:311-318.

Hucka, M., Finney, A., Sauro, H. M., Bolouri, H., Doyle, J. C., Kitano, H., Arkin, A. P., Bornstein, B. J., Bray, D., Cornish-Bowden, A., Cuellar, A. A., Dronov, S., Gilles, E. D., Ginkel, M., Gor, V., Goryanin, I. I., Hedley, W. J., Hodgman, T. C., Hofmeyr, J. H., Hunter, P. J., Juty, N. S. Kasberger, J. L., Kremling, A., Kummer, U., Le Novère, N., Loew, L. M., Lucio, D., Mendes, P., Minch, E., Mjolsness, E. D., Nakayama, Y., Nelson, M. R., Nielsen, P. F., Sakurada, T., Schaff, J. C., Shapiro, B. E., Shimizu, T. S., Spence, H. D., Stelling, J., Takahashi, K., Tomita, M. Wagner, J., Wang, J., and SBML Forum. 2003. The systems biology markup language (SBML): A medium for representation and exchange of biochemical network models. Bioinformatics 19:524-531.

Huot, B., Castroverde, C. D. M., Velásquez, A. C., Hubbard, E., Pulman, J. A., Yao, J., Childs, K. L., Tsuda, K., Montgomery, B. L., and He, S. Y. 2017. Dual impact of elevated temperature on plant defence and bacterial virulence in Arabidopsis. Nat. Commun. 8: Article 1808.

Jiang, X., Zhang, H., Quan, X., Liu, Z., and Yin, Y. 2017. Disease-related gene module detection based on a multi-label propagation clustering algorithm. PLoS One 12:e0178006.

Jiang, Z., Dong, X., Li, Z. G., He, F., and Zhang, Z. 2016. Differential coexpression analysis reveals extensive rewiring of Arabidopsis gene coexpression in response to Pseudomonas syringae Infection. Sci. Rep. 6: Article 35064

Kamoun, S., Furzer, O., Jones, J. D., Judelson, H. S., Ali, G. S., Dalio, R. J., Roy, S. G., Schena, L., Zambounis, A., Panabières, F., Cahill, D., Ruocco, M., Figueiredo, A., Chen, X. R., Hulvey, J., Stam, R., Lamour, K., Gijzen, M., Tyler, B. M., Grünwald, N. J., Mukhtar, M. S., Tomé, D. F., Tör, M., Van Den Ackerveken, G., McDowell, J., Daayf, F., Fry, W. E., Lindqvist-Kreuze, H., Meijer, H. J., Petre, B., Ristaino, J., Yoshida, K., Birch, P. R., and Govers, F. 2015. The Top 10 oomycete pathogens in molecular plant pathology. Mol. Plant Pathol. 16:413-434

Karlić, R., Chung, H. R., Lasserre, J., Vlahovicek, K., and Vingron, M 2010. Histone modification levels are predictive for gene expression. Proc. Natl. Acad. Sci. U.S.A. 107:2926-2931.

Karr, J. R., Phillips, N. C., and Covert, M. W. 2014. WholeCellSimDB: A hybrid relational/HDF database for whole-cell model predictions. Database 2014:bau095.

Karr, J. R., Sanghvi, J. C., Macklin, D. N., Gutschow, M. V., Jacobs, J. M., Bolival, B., Jr., Assad-Garcia, N., Glass, J. I., and Covert, M. W. 2012. A whole-cell computational model predicts phenotype from genotype. Cell 150:389-401.

Kurubanjerdjit, N., Huang, C.-H., Lee, Y. L., Tsai, J. J., and Ng, K.-L. 2013. Prediction of microRNA-regulated protein interaction pathways in Arabidopsis using machine learning algorithms. Comput. Biol. Med. 43:1645-1652.

Kushwaha, S. K., Chauhan, P., Hedlund, K., and Ahrén, D. 2016. NBSPred A support vector machine-based high-throughput pipeline for plan resistance protein NBSLRR prediction. Bioinformatics 32:12231225 .

Le Novère, N. 2015. Quantitative and logic modelling of molecular and gene networks. Nat. Rev. Genet. 16:146-158.

Le Novère, N., Finney, A., Hucka, M., Bhalla, U. S., Campagne, F., Collado-Vides, J., Crampin, E. J., Halstead, M., Klipp, E., Mendes, P. Nielsen, P., Sauro, H., Shapiro, B., Snoep, J. L., Spence, H. D., and Wanner, B. L. 2005. Minimum information requested in the annotation of biochemical models (MIRIAM). Nat. Biotechnol. 23:1509-1515. 
Leal, L. G., López, C., and López-Kleine, L. 2014. Construction and comparison of gene co-expression networks shows complex plant immune responses. PeerJ 2:e610.

LeCun, Y., Bengio, Y., and Hinton, G. 2015. Deep learning. Nature 521: 436-444.

Li, M., Zhang, J., Liu, Q., Wang, J., and Wu, F.-X. 2014. Prediction of disease-related genes based on weighted tissue-specific networks by using DNA methylation. BMC Med. Genomics 7:S4.

Liu, J., Hua, P., Hui, L., Zhang, L. L., Hu, Z., and Zhu, Y. W. 2016. Identification of hub genes and pathways associated with hepatocellular carcinoma based on network strategy. Exp. Ther. Med. 12:2109-2119.

Lopez, J. A., Sun, Y., Blair, P. B., and Mukhtar, M. S. 2015. TCP three-way handshake: Linking developmental processes with plant immunity. Trends Plant Sci. 20:238-245.

Lovelace, A. H., Smith, A., and Kvitko, B. H. 2018. Pattern-triggered immunity alters the transcriptional regulation of virulence-associated genes and induces the sulfur starvation response in Pseudomonas syringae pv. tomato DC3000. Mol. Plant-Microbe Interact. 31:750-765.

Lü, L., Chen, D., Ren, X.-L., Zhang, Q.-M., Zhang, Y.-C., and Zhou, T. 2016a. Vital nodes identification in complex networks. Phys. Rep. 650: 1-63.

Lü, L., Zhou, T., Zhang, Q. M., and Stanley, H. E. 2016b. The H-index of a network node and its relation to degree and coreness. Nat. Commun. 7: Article 10168.

Ma, B., Charkowski, A. O., Glasner, J. D., and Perna, N. T. 2014. Identification of host-microbe interaction factors in the genomes of soft rot-associated pathogens Dickeya dadantii 3937 and Pectobacterium carotovorum WPP14 with supervised machine learning. BMC Genomics 15:508.

Masalia, R. R., Bewick, A. J., and Burke, J. M. 2017. Connectivity in gene coexpression networks negatively correlates with rates of molecular evolution in flowering plants. PLoS One 12:e0182289.

McCormack, M. E., Lopez, J. A., Crocker, T. A., and Mukhtar, M. S. 2016. Making the right connections: Network biology and plant immune system dynamics. Curr. Plant Biol. 5:2-12.

McMurray, B., and Hollich, G. 2009. Core computational principles of language acquisition: Can statistical learning do the job? Introduction to special section. Dev. Sci. 12:365-368.

Mehra, L. K., Cowger, C., Gross, K., and Ojiambo, P. S. 2016. Predicting pre-planting risk of Stagonospora nodorum blotch in winter wheat using machine learning models. Front. Plant Sci. 7:390.

Meshkin, A., Shakery, A., and Masoudi-Nejad, A. GPS: Identification of disease genes by rank aggregation of multi-genomic scoring schemes. Genomics In press. doi:10.1016/j.ygeno.2018.03.017

Mishra, B., Sun, Y., Ahmed, H., Liu, X., and Mukhtar, M. S. 2017. Global temporal dynamic landscape of pathogen-mediated subversion of Arabidopsis innate immunity. Sci. Rep. 7: Article 7849.

Mukhtar, M. S. 2013. Engineering NLR immune receptors for broadspectrum disease resistance. Trends Plant Sci. 18:469-472.

Mukhtar, M. S., Carvunis, A. R., Dreze, M., Epple, P., Steinbrenner, J., Moore, J., Tasan, M., Galli, M., Hao, T., Nishimura, M. T., Pevzner, S. J., Donovan, S. E., Ghamsari, L., Santhanam, B., Romero, V., Poulin, M. M., Gebreab, F., Gutierrez, B. J., Tam, S., Monachello, D., Boxem, M., Harbort, C. J., McDonald, N., Gai, L., Chen, H., He, Y., European Union Effectoromics Consortium, Vandenhaute, J., Roth, F. P., Hill, D. E., Ecker, J. R., Vidal, M., Beynon, J., Braun, P., and Dangl, J. L. 2011. Independently evolved virulence effectors converge onto hubs in a plant immune system network. Science 333:596-601.

Mukhtar, M. S., McCormack, M. E., Argueso, C. T., and PajerowskaMukhtar, K. M. 2016. Pathogen tactics to manipulate plant cell death. Curr. Biol. 26:R608-R619.

Nobori, T., Mine, A., and Tsuda, K. 2018a. Molecular networks in plantpathogen holobiont. FEBS Lett. 592:1937-1953.

Nobori, T., Velásquez, A. C., Wu, J., Kvitko, B. H., Kremer, J. M., Wang, Y., He, S. Y., and Tsuda, K. 2018b. Transcriptome landscape of a bacterial pathogen under plant immunity. Proc. Natl. Acad. Sci. U.S.A. 115: E3055-E3064.

Ohler, U., Liao, G.-C., Niemann, H., and Rubin, G. M. 2002. Computational analysis of core promoters in the Drosophila genome. Genome Biol. 3:research0087.

Ouyang, Z., Zhou, Q., and Wong, W. H. 2009. ChIP-Seq of transcription factors predicts absolute and differential gene expression in embryonic stem cells. Proc. Natl. Acad. Sci. U.S.A. 106:21521-21526.

Pajerowska-Mukhtar, K. M., Emerine, D. K., and Mukhtar, M. S. 2013. Tell me more: Roles of NPRs in plant immunity. Trends Plant Sci. 18:402-411.

Pan, A., Lahiri, C., Rajendiran, A., and Shanmugham, B. 2016. Computational analysis of protein interaction networks for infectious diseases. Brief. Bioinf. 17:517-526.
Pei, S., Muchnik, L., Andrade, J. S., Jr., Zheng, Z., and Makse, H. A. 2014. Searching for superspreaders of information in real-world social media. Sci. Rep. 4: Article 5547.

Pirooznia, M., Yang, J. Y., Yang, M. Q., and Deng, Y. 2008. A comparative study of different machine learning methods on microarray gene expression data. BMC Genomics 9:S13.

Pritchard, L., and Birch, P. R. J. 2014. The zigzag model of plant-microbe interactions: Is it time to move on? Mol. Plant Pathol. 15:865-870.

Qiang, X., Kou, Z., Fang, G., and Wang, Y. 2018. Scoring amino acid mutations to predict avian-to-human transmission of avian influenza viruses. Molecules 23:1584.

Ramadan, E., Alinsaif, S., and Hassan, M. R. 2016. Network topology measures for identifying disease-gene association in breast cancer. BMC Bioinf. 17:274.

Rangarajan, N., Kulkarni, P., and Hannenhalli, S. 2015. Evolutionarily conserved network properties of intrinsically disordered proteins. PLoS One 10:e0126729.

Schatschneider, S., Persicke, M., Watt, S. A., Hublik, G., Pühler, A., Niehaus, K., and Vorhölter, F. J. 2013. Establishment, in silico analysis, and experimental verification of a large-scale metabolic network of the xanthan producing Xanthomonas campestris pv. campestris strain B100. J. Biotechnol. 167:123-134.

Schmidhuber, J. 2015. Deep learning in neural networks: An overview. Neural Netw. 61:85-117.

Serin, E. A., Nijveen, H., Hilhorst, H. W., and Ligterink, W. 2016. Learning from co-expression networks: Possibilities and challenges. Front. Plant Sci. 7:444.

Smakowska-Luzan, E., Mott, G. A., Parys, K., Stegmann, M., Howton, T. C., Layeghifard, M., Neuhold, J., Lehner, A., Kong, J., Grünwald, K., Weinberger, N., Satbhai, S. B., Mayer, D., Busch, W., Madalinski, M., Stolt-Bergner, P., Provart, N. J., Mukhtar, M. S., Zipfel, C., Desveaux, D., Guttman, D. S., and Belkhadir, Y. 2018. An extracellular network of Arabidopsis leucine-rich repeat receptor kinases. Nature 553:342-346.

Smallbone, K., Simeonidis, E., Swainston, N., and Mendes, P. 2010. Towards a genome-scale kinetic model of cellular metabolism. BMC Syst. Biol. 4:6.

Sperschneider, J., Dodds, P. N., Singh, K. B., and Taylor, J. M. 2018 ApoplastP: Prediction of effectors and plant proteins in the apoplast using machine learning. New Phytol. 217:1764-1778.

Sperschneider, J., Gardiner, D. M., Dodds, P. N., Tini, F., Covarelli, L., Singh, K. B., Manners, J. M., and Taylor, J. M. 2016. EffectorP: Predicting fungal effector proteins from secretomes using machine learning. New Phytol. 210:743-761.

Toju, H., Tanabe, A. S., and Sato, H. 2018. Network hubs in root-associated fungal metacommunities. Microbiome 6:116.

Toju, H., Yamamoto, S., Tanabe, A. S., Hayakawa, T., and Ishii, H. S. 2016. Network modules and hubs in plant-root fungal biomes. J. R. Soc. Interface 13:20151097.

Tsuda, K. 2018. Division of tasks: Defense by the spatial separation of antagonistic hormone activities. Plant Cell Physiol. 59:3-4.

Tully, J. P., Hill, A. E., Ahmed, H. M., Whitley, R., Skjellum, A., and Mukhtar, M. S. 2014. Expression-based network biology identifies immune-related functional modules involved in plant defense. BMC Genomics 15:421.

Viljanen, N., Honkavaara, E., Näsi, R., Hakala, T., Niemeläinen, O., and Kaivosoja, J. 2018. A novel machine learning method for estimating biomass of grass swards using a photogrammetric canopy height model, images and vegetation indices captured by a drone. Agriculture 8:70.

Waltemath, D., Adams, R., Beard, D. A., Bergmann, F. T., Bhalla, U. S., Britten, R., Chelliah, V., Cooling, M. T., Cooper, J., Crampin, E. J., Garny, A., Hoops, S., Hucka, M., Hunter, P., Klipp, E., Laibe, C., Miller, A. K., Moraru, I., Nickerson, D., Nielsen, P., Nikolski, M., Sahle, S., Sauro, H. M., Schmidt, H., Snoep, J. L., Tolle, D., Wolkenhauer, O., and Le Novère, N. 2011b. Minimum information about a simulation experiment (MIASE). PLOS Comput. Biol. 7:e1001122.

Waltemath, D., Adams, R., Bergmann, F. T., Hucka, M., Kolpakov, F., Miller, A. K., Moraru, I. I., Nickerson, D., Sahle, S., Snoep, J. L., and Le Novère, N. 2011a. Reproducible computational biology experiments with SED-ML-The simulation experiment description markup language. BMC Syst. Biol. 5:198.

Wang, J.-Y., Yao, W.-X., Wang, Y., Fan, Y. L., and Wu, J.-B. 2017. Network analysis reveals crosstalk between autophagy genes and disease genes. Sci. Rep. 7: Article 44391.

Wang, S., Peng, J., Ma, J., and Xu, J. 2016. Protein secondary structure prediction using deep convolutional neural fields. Sci. Rep. 6: Article 18962.

Wang, X., Ma, J., Li, X., Zhao, X., Lin, Z., Chen, J., and Shao, Z. 2015. Optimization of chemical fungicide combinations targeting the maize 
fungal pathogen, Bipolaris maydis: A systematic quantitative approach. IEEE Trans. Biomed. Eng. 62:80-87.

Washington, E. J., Mukhtar, M. S., Finkel, O. M., Wan, L., Banfield, M. J., Kieber, J. J., and Dangl, J. L. 2016. Pseudomonas syringae type III effector HopAF1 suppresses plant immunity by targeting methionine recycling to block ethylene induction. Proc. Natl. Acad. Sci. U.S.A. 113: E3577-E3586.

Weßling, R., Epple, P., Altmann, S., He, Y., Yang, L., Henz, S. R., McDonald, N., Wiley, K., Bader, K. C., Gläßer, C., Mukhtar, M. S., Haigis, S., Ghamsari, L., Stephens, A. E., Ecker, J. R., Vidal, M., Jones, J. D., Mayer, K. F., Ver Loren van Themaat, E., Weigel, D., SchulzeLefert, P., Dangl, J. L., Panstruga, R., and Braun, P. 2014. Convergent targeting of a common host protein-network by pathogen effectors from three kingdoms of life. Cell Host Microbe 16:364-375.

Westermann, A. J., Gorski, S. A., and Vogel, J. 2012. Dual RNA-seq of pathogen and host. Nat. Rev. Microbiol. 10:618-630.
Willett, D. S., George, J., Willett, N. S., Stelinski, L. L., and Lapointe, S. L. 2016. Machine learning for characterization of insect vector feeding. PLOS Comput. Biol. 12:e1005158.

Wójcik, P. I., Ouellet, T., Balcerzak, M., and Dzwinel, W. 2015. Identification of biomarker genes for resistance to a pathogen by a novel method for meta-analysis of single-channel microarray datasets. J. Bioinf. Comput. Biol. 13:1550013.

Wolf, T., Kämmer, P., Brunke, S., and Linde, J. 2018. Two's company: Studying interspecies relationships with dual RNA-seq. Curr. Opin. Microbiol. 42:7-12.

Yu, H., Kim, P. M., Sprecher, E., Trifonov, V., and Gerstein, M. 2007. The importance of bottlenecks in protein networks: Correlation with gene essentiality and expression dynamics. PLOS Comput. Biol. 3:e59.

Zhao, J., Yang, T. H., Huang, Y., and Holme, P. 2011. Ranking candidate disease genes from gene expression and protein interaction: A Katzcentrality based approach. PLoS One 6:e24306. 